\title{
Successful pregnancy outcome with the levonorgestrel-releasing intrauterine system
}

\author{
Andrea Pilkington, Nasira Misfar, David W Polson
}

\section{Introduction}

The levonorgestrel-releasing intrauterine system (IUS) is extremely effective, with pregnancy rates comparable to tubal ligation (i.e. a 5-year cumulative rate varying from 0.5 to 1.1). ${ }^{1}$ Women using the device should be fully counselled about the risks of failure and the risks of ectopic pregnancy, although those using an IUS are actually at a much lower risk of ectopic pregnancy than those not using any contraception at all. ${ }^{1}$

An ongoing intrauterine pregnancy with the IUS is very rare. One report confirmed that of 678 insertions only five pregnancies occurred, none of which proceeded to term with the IUS in situ. ${ }^{2}$ This case report describes an ongoing pregnancy with a IUS in situ.

\section{Case report}

A 30-year-old woman, para 2, had an IUS inserted on 17 November 2005 on account of heavy and irregular periods after the birth of her second child 15 weeks earlier. The date of her last menstrual period was 31 October 2005 and therefore the IUS was inserted on Day 18 of the cycle. The only unprotected intercourse she recalls is an occasion where a condom had split prior to insertion of the IUS.

A positive pregnancy test result on 10 December 2005 resulted in the patient presenting to the gynaecology ward as an urgent referral. An ultrasound scan performed on 15 December 2005 confirmed a viable 6.5-week intrauterine pregnancy (crown-rump length, $6 \mathrm{~mm}$ ) with the IUS seen below the gestation sac. This confirmed that conception must have occurred prior to insertion of the IUS, sometime between 10 and 14 November. The patient was advised to have the IUS removed because of possible adverse effects on the pregnancy. This was not possible, however, as the IUS threads were not visible.

Two weeks later a repeat scan confirmed an ongoing pregnancy and the patient was counselled about the potential risks of continuing with the pregnancy, including miscarriage, premature delivery and preterm rupture of membranes. Other risks discussed included potential masculinisation of the fetus by the levonorgestrel. Termination was discussed but the patient wished to continue with the pregnancy.

At 16 weeks the patient was seen on the Maternity Day Case Unit with vaginal spotting. No threads were seen on examination and the patient was reassured and allowed to go home. No further bleeding occurred. A detailed ultrasound scan at 20 weeks showed a normal fetus.

J Fam Plann Reprod Health Care 2008; 34(1): 60 (Accepted 2 May 2007)

\section{Department of Obstetrics and Gynaecology, Hope Hospital,}

Salford, UK

Andrea Pilkington, MBChB, Senior House Officer

Nasira Misfar, MBBS, Registrar

David W Polson, MD, FRCOG, Consultant

Correspondence: Dr Andrea Pilkington, Department of

Obstetrics and Gynaecology, Hope Hospital, Salford M6 8HD, UK.

E-mail: andreapilkington2@hotmail.com
The patient went on to have a normal and uneventful delivery at term of a live male infant with no abnormalities. The IUS was recovered from the placenta at delivery.

\section{Discussion}

The IUS is used for its contraceptive benefits and also in the treatment of menorrhagia. Most women experience reduced menstrual flow after insertion with some achieving amenorrhoea, commonly after the first year of use. ${ }^{3}$

Follow-up studies have indicated that the failure rate of the IUS is extremely low, with $0.033 \%$ as the total and $0.008 \%$ as the true failure rates, respectively. ${ }^{1,4}$ The latest reported studies 1,4 indicate that $62.5 \%$ of pregnancies reported after IUS insertion were ectopic whilst $37.5 \%$ were intrauterine. Of the 15 intrauterine pregnancies, five were terminated and eight of the remainder miscarried. Only two pregnancies were reported to proceed to term.

Most pregnancies occur in the first 15 months following insertion and are due to an unnoticed expulsion (expulsion rate of 6.4 per 100 women at 12 months) ${ }^{2}$ In the present case the IUS was inserted between 4 and 7 days after ovulation and therefore should not be considered a failure of the system.

This case report clearly demonstrates that all patients with an irregular cycle should be offered a pregnancy test prior to IUS insertion and that insertion should be timed at the beginning of the cycle, as recommended by the manufacturer. If the IUS is inserted at any other time in the cycle then effective contraception cannot be guaranteed. Evidence has confirmed that the majority of pregnancies conceived prior to the time of insertion will miscarry, but in this rare case the pregnancy did continue uneventfully to term.

As the fetus was male, no effects of masculinisation of a female fetus can be commented upon, although this is a theoretical possibility since it has been reported with the use of oral progestogens in early pregnancy. 5 Consequently, although many women may opt for a termination and up to $80 \%$ may miscarry, this case report demonstrates that a normal infant can be delivered at term with an IUS in situ.

\section{Statements on funding and competing interests}

Funding None identified.

Competing interests None identified.

\section{References}

1 Backman T, Rauramo I, Huhtala S, Koskenvuo M. Pregnancy during the use of levonorgestrel intrauterine system. $A m \mathrm{~J}$ Obstet Gynecol 2004; 190: 50-54.

2 Cox M, Tripp J, Blacksell S. Clinical performance of the levonorgestrel intrauterine system in routine use by the UK Family Planning and Reproductive Health Research Network: 5-year report. Br J Fam Plann 2002; 28: 73-77.

3 Sturridge F, Guillebaud J. A risk-benefit assessment of the levonorgestrel-releasing intrauterine system. Drug Saf 1996; 15: $430-440$.

4 Backman T, Huhtala S, Tuominen J, Luoto R, Erkkola R, Blom $\mathrm{T}$, et al. Sixty thousand woman-years of experience on the levonorgestrel intrauterine system: an epidemiological survey in Finland. Eur J Contracept Reprod Health Care 2001; 6(Suppl. 1): 23-26.

5 Herbst AL. Exogenous hormones in pregnancy. Clin Obstet Gynecol 1973; 16: 37-50. 